\title{
EYE OF TIGER SIGN: CLINICAL PHENOTYPES
}

\section{G. Sree Ranga Lakshmi1 Archana $^{2}$, R. Ramesh ${ }^{3}$, K. Kiran Kumar 4 , P. Vijayalakshmi 5 , M. Deepti Kiran 6}

${ }^{1}$ Associate Professor, Department of Neurology, Osmania General Hospital and College, Hyderabad, Telangana. ${ }^{2}$ Assistant Professor, Department of Neurology, Osmania General Hospital and College, Hyderabad, Telangana. ${ }^{3}$ Assistant Professor, Department of Neurology, Osmania General Hospital and College, Hyderabad, Telangana. ${ }^{4}$ DM Post Graduate, Department of Neurology, Osmania General Hospital and College, Hyderabad, Telangana. ${ }^{5}$ DM Post Graduate, Department of Neurology, Osmania General Hospital and College, Hyderabad, Telangana. ${ }^{6}$ DM Post Graduate, Department of Neurology, Osmania General Hospital and College, Hyderabad, Telangana.

\section{ABSTRACT}

The Eye-of-the-Tiger sign is a radiological sign that refers to abnormal low signal intensity in the globus pallidus with a central longitudinal zone of high signal as seen on T2-weighted MRI images. The sign is considered the distinctive radiologic feature of Neurodegeneration with Brain Iron Accumulation (NBIA) type 1, also known as Pantothenate Kinase Associated Neurodegeneration (PKAN). NBIA is a group of inherited neurologic disorders, in which iron accumulates in the basal ganglia resulting in progressive extrapyramidal symptoms. Clinical features and severity may vary with the age of onset and the nature of the underlying mutation. PKAN can present as early onset and late onset forms. We report a few case studies with varying clinical phenotypes and showing imaging features of eye of tiger. Two patients did not develop the characteristic features of PKAN even after five years of follow-up.

\section{KEYWORDS}

Eye-of-Tiger Sign, NBIA, PKAN, Extrapyramidal, Stimming, Caudate Atrophy.

HOW TO CITE THIS ARTICLE: Lakshmi GSR, Archana, Ramesh R, et al. Eye of tiger sign: clinical phenotypes. J. Evolution Med. Dent. Sci. 2016;5(60):4190-4193, DOI: 10.14260/jemds/2016/956

\section{INTRODUCTION}

The Eye-of-the-Tiger sign is a well-known radiological sign, which refers to low-signal intensity rings surrounding the central high signal intensity regions in the medial aspect of globus pallidi on T2-weighted MRI. The surrounding hypointensity of the globus pallidus is due to excess iron accumulation. The central hyperintensity is possibly due to gliosis.(1) The sign is considered as the distinctive radiologic feature of Neurodegeneration with Brain Iron Accumulation (NBIA) type 1, also known as Pantothenate Kinase Associated Neurodegeneration (PKAN). NBIA encompasses a group of disorders, which have some common features like presentation in paediatric or early adulthood with extrapyramidal and cerebellar signs and corresponding abnormalities in the basal ganglia and cerebellum on neuroimaging, clinically heterogeneous, refractory to therapy, relentlessly progressive and inevitably fatal. From time to time, novel clinical presentations of NBIA are being reported. Here, we report a short series of patients with Eye-of-Tiger sign with variety of clinical manifestations.

\section{CASE 1}

A 32-year female presented with 4 years history of altered behaviour, depression, bradykinesia, reduced level of social and occupational functioning.

On examination, there was mild memory loss and rigidity of all four limbs (more on the right side) and right-sided striatal toe with rapid deterioration in the last 3 months to mute and rigid and disoriented state. She was a product of non-consanguineous parentage and there was no family

Financial or Other, Competing Interest: None.

Submission 05-07-2016, Peer Review 18-07-2016,

Acceptance 20-07-2016, Published 28-07-2016.

Corresponding Author:

Dr. G. Sree Ranga Lakshmi,

Associate Professor,

Department of Neurology,

Osmania General Hospital,

Hyderabad, Telangana.

E-mail: rangalakshmi2000@gmail.com

DOI: $10.14260 /$ jemds/2016/956 history of similar illness. MRI brain revealed Eye-of-the-Tiger sign with caudate atrophy (Fig. 1).

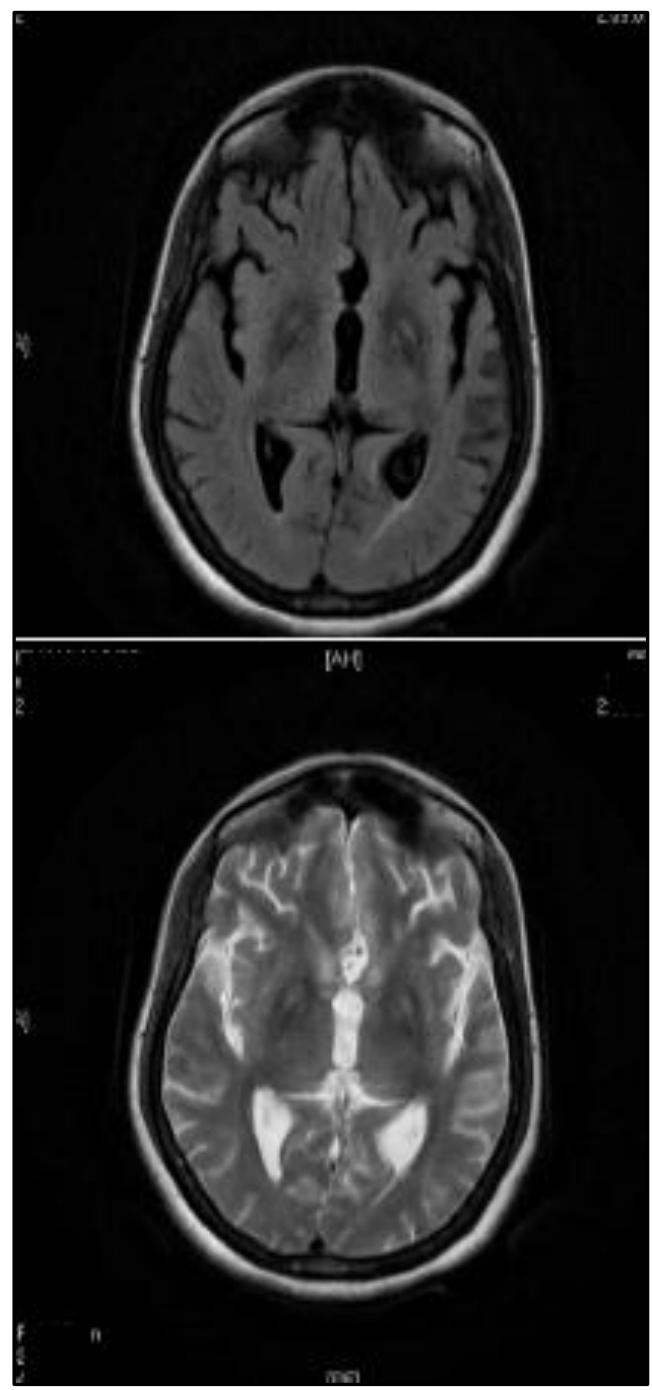




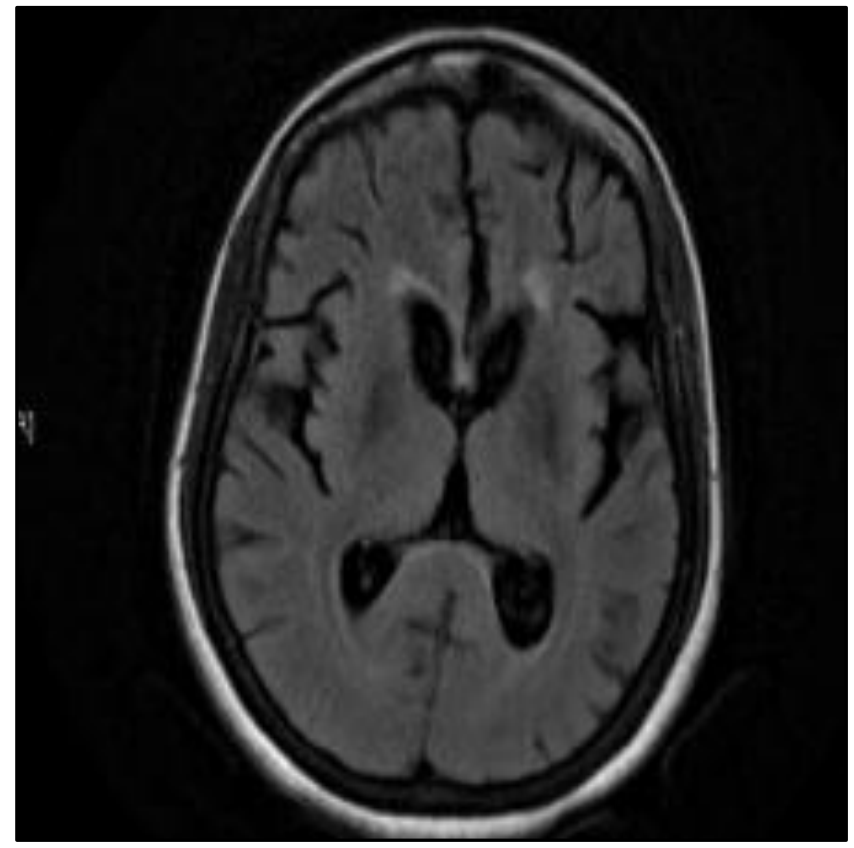

Fig. 1: FLAIR and T2 Axial MRI Brain Images showing Eye of Tiger Sign and Caudate Atrophy

\section{CASE 2}

A 17-year male, symptomatic since 4 years presented with stimming other peoples' clothes. He was getting low grades since he started schooling. He was born out of consanguineous parentage. His serum iron profile was normal. MRI brain showed classic Eye-of-the-Tiger sign (Fig. 2).
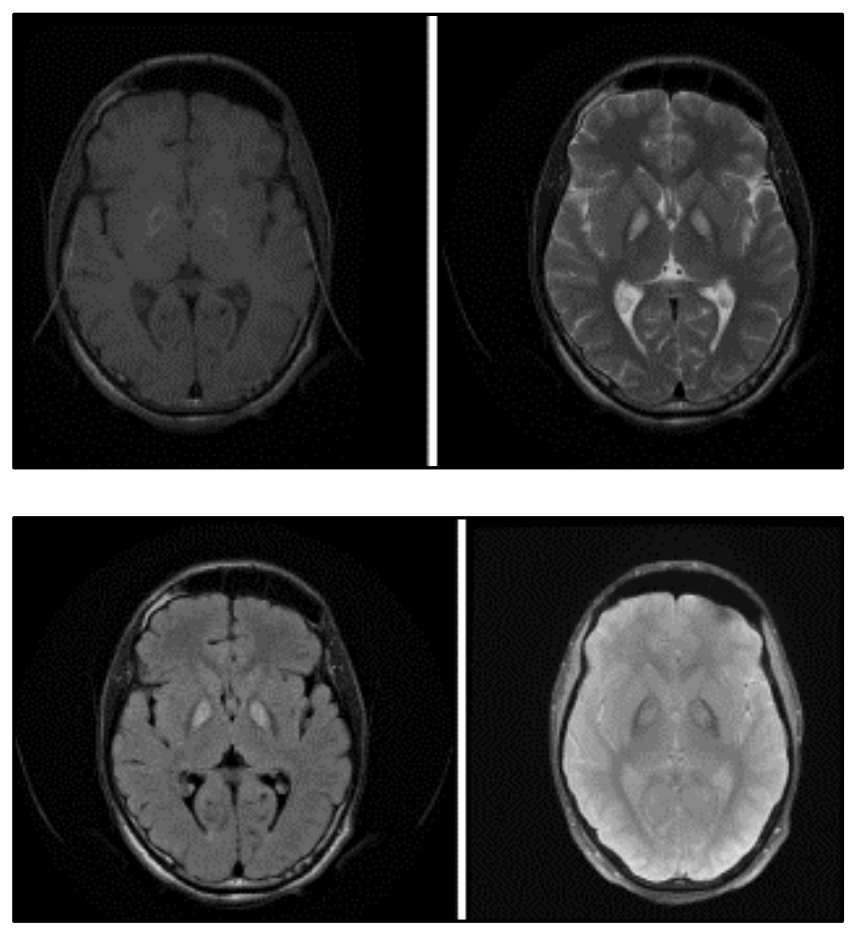

Fig. 2: T1, T2 FLAIR and Gradient Echo Images showing Eye of Tiger Sign

\section{CASE 3}

A 13-year boy, brother of previous case was symptomatic since 6 months, presented with stimming other peoples' clothes with history of having low grades since he started schooling.
Both of them did not show any autistic features. Examination did not reveal $\mathrm{KF}$ ring or any extra pyramidal features like dystonia or any neurological deficit. MRI brain showed classic Eye-of-the-Tiger sign (Fig. 3).

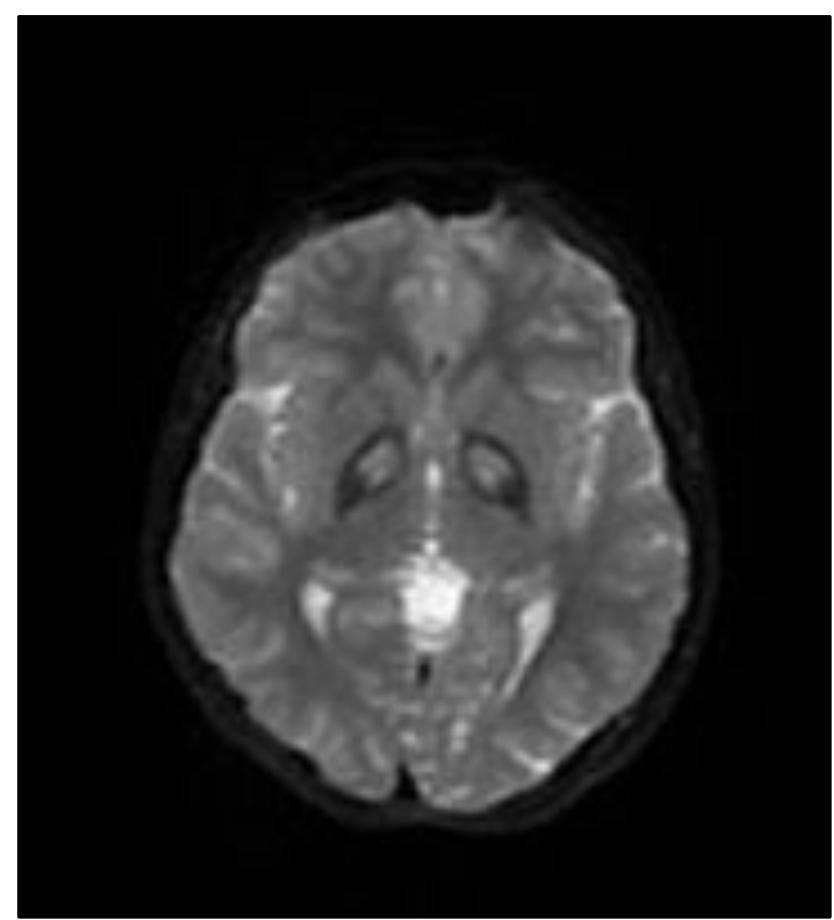

Fig. 3: T2 WI Image showing Eye of Tiger Sign

CASE 4

A 60-year male, father of the above two children, asymptomatic and his neurological examination was normal. MRI brain revealed T2 hypointensity in globus pallidus with subtle central hyperintensity, which is suggestive of excess iron deposition in basal ganglia (Fig. 4).

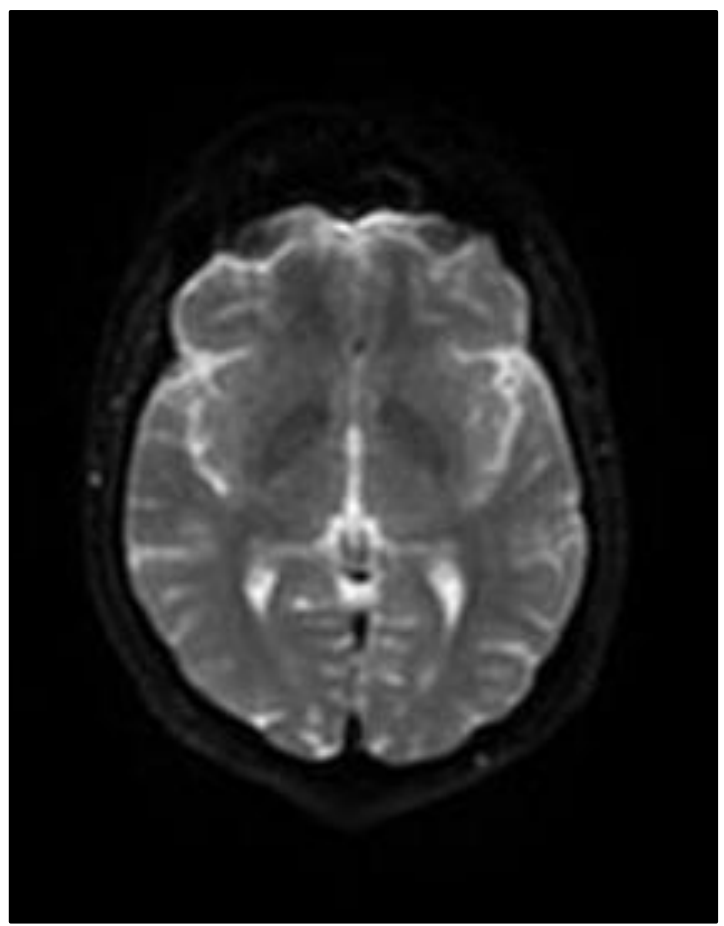

Fig. 4: T2 WI Image showing Hypointensity in Globus Pallidus with Subtle Central Hyperintensity 


\section{CASE 5}

A 31-year female, symptomatic since 7 yrs. presented with falls, inappropriate laughter and rigidity of lower limbs, which progressed gradually and lead to a bedridden state. She was the first cousin of previous children. Her MRI brain also revealed classical Eye-of-the-Tiger sign (Fig. 5).

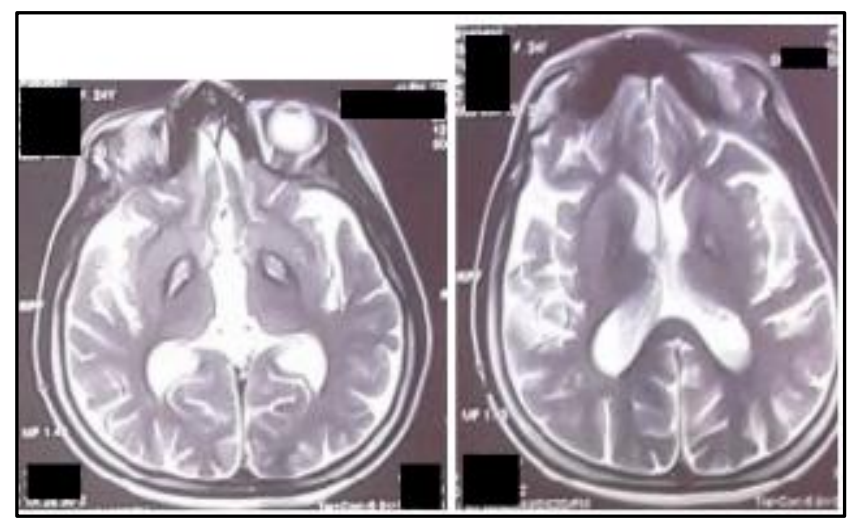

Fig. 5: T2 WI Image showing Eye of Tiger Sign

\section{DISCUSSION}

The name "Eye-of-the-Tiger" sign was coined by Sethi et al(2) in 1988, which is a radiological sign that refers to abnormal low signal intensity in the globus pallidus with a central longitudinal zone of high signal as seen on T2 weighted MRI images. They described this sign in two female patients who presented with blepherospasm and dystonia respectively. In the transverse plane through the basal ganglia, this appears as a tiger like image with prominent eyes. The sign is considered the distinctive radiologic feature of Neurodegeneration with Brain Iron Accumulation (NBIA) type 1, also known as pantothenate kinase associated neurodegeneration (PKAN). All the disorders of NBIA have iron deposition in the globus pallidus, but differ in of other features. But they are all characterized by occurrence of a movement disorder and progressive neurodegenerative course. Some of the NBIA disorders include aceruloplasminemia, fatty acid hydroxylase-associated neurodegeneration, infantile neuroaxonal dystrophy, KuforRakeb syndrome, neuroaxonal dystrophy, neuroferritinopathy. Except neuroferritinopathy, all the other are autosomal recessive in inheritance.(3)

The disease onset is variable and may range from early childhood to old age. Clinical features and severity may vary with the age of onset and the nature of the underlying mutation. Iron deposition also occurs to a lesser degree in multiple sclerosis human immunodeficiency virus dementia, Friedreich's ataxia and Alzheimer and Parkinson diseases.(3) In classic PKAN the symptoms usually start to appear before age 6 on average around age 3 with profound dystonia, dysarthria, spasticity and pyramidal tract signs and pigmentary retinopathy leading to night blindness and visual field constriction. Intellectual deterioration is variable and tends to be more severe in those with earlier disease onset. $(4,5)$ Atypical forms have later age of onset with dystonia often manifesting as prominent orobuccolingual actioninduced eating dystonia. Parkinsonism and prominent neuropsychiatric features such as hyperactivity, impulsivity, depression, obsessive-compulsive disorder and vocal and motor tics can be seen.
Our first patient presented at the age of 28 years with behaviour abnormalities and parkinsonian features. She had the onset at later age and progressed rapidly in three months, became mute and disoriented. Her imaging showed in addition to Eye of Tiger Sign, bilateral caudate atrophy. Genetic testing for CAG repeats did not show any expansion, thus excluding Huntington's disease. Cortical and cerebellar atrophy is described in certain forms of NBIA.(6), but till now no case is reported with caudate trophy.

The other male patients belong to the same family. (Father and sons). Both the children were having poor scholastic performance. They presented with the same complaint of smelling the other persons and clothes, which is called stimming. This is observed in autistic disorders, though $10 \%$ of non-autistic children can have this.

Both of them did not have any other extrapyramidal or other behavioural disturbances except stimming till the last follow-up (7 years in first child and 2 years in second child. The father is asymptomatic). Their MRI brain showed Eye-ofTiger sign. Literature search did not show similar imaging findings in autistic disorders.

Their father was totally asymptomatic and his MRI brain (was done out of curiosity) showed bilateral T2 hypointensity in globus pallidus with subtle central hyperintensity.

The biochemical workup including serum iron, ferritin, copper, ceruloplasmin levels, hepatic and renal function tests were normal. There was no KF ring.

The last patient who was the first cousin of above children was symptomatic in early twenties with extrapyramidal and behaviour disturbances and now she is bedridden. Her MRI revealed classical Eye-of-Tiger sign.

We could not do the mutational study of the gene PANK2, as it is not available in our country. There are few PANK2 negative Eye-of-the-Tiger sign cases being reported.(7)

Till now there was only one case report describing Eyeof-the-Tiger sign in a 48-year healthy adult.(8) Ours is the second report showing this sign in three people belonging to same family without any extrapyramidal features. These are under follow-up and did not develop any other neurological signs till now.

\section{CONCLUSION}

The degree of iron deposition correlates incompletely with clinical symptoms in some individuals. Though iron is a useful neuroimaging marker in NBIA, it may not be necessary nor sufficient to produce the disease phenotype.

\section{REFERENCES}

1. Chang CL, Lin CM. Eye-of-the-Tiger sign is not pathognomonic of pantothenate kinase-associated neurodegeneration in adult cases. Brain Behav 2011;1(1):55-6.

2. Sethi KD, Adams RJ, Loring DW, et al. Hallervordenspatz syndrome: clinical and magnetic resonance imaging correlations. Ann Neurol 1988;24(5):692-4.

3. Kruer MC, Boddaert $\mathrm{N}$, Schneider SA, et al. Neuroimaging features of neurodegeneration with brain iron accumulation. AJNR Am J Neuroradiol 2012;33(3):407-14.

4. Hayflick SJ. Pantothenate kinase-associated neurodegeneration (formerly Hallervorden-Spatz syndrome). J Neurol Sci 2003;207(1-2):106-7. 
5. Hayflick SJ, Westaway SK, Levinson B, et al. Genetic, clinical, and radiographic delineation of HallervordenSpatz syndrome. Engl J Med 2003;348(1):33-40.

6. Gregory A, Hayflick S. Neurodegeneration with brain iron accumulation disorders overview 2013. In: Pagon RA, Adam MP, Ardinger HH, et al. (edi) GeneReviews ${ }^{\circledR}$ [Internet]. Seattle (WA): University of Washington, Seattle 1993-2016. Available from:

http://www.ncbi.nlm.nih.gov/books/NBK121988/
7. Hartig MB, Hörtnagel K, Garavaglia B, et al. Genotypic and phenotypic spectrum of PANK 2 mutations in patients with neurodegeneration with brain iron accumulation. Ann Neurol 2006;59(2):248-56.

8. Bogaard VSJ, Kruit MC, Dumas EM, et al. Eye-of-theTiger sign in a 48 year healthy adult. J Neurol Sci 2014;336(1-2):254-6. 